\title{
UTILIDAD DE LA LAPAROSCOPIA EN GINECOLOGIA
}

\author{
Dr. Luis Armando Muñoz ${ }^{*}$, M.D.
}

\section{Definición :}

Es el examen endoscópico de la cavidad peritoneal por medio de un tubo o Laparoscopio a través de la pared anterior del abdomen. Se le conoce además con los siguientes nombres: Peritoneoscopia, Celioscopia.

\section{Historia :}

Kelling de la ciudad de Dresde en 1901, mediante el Citoscopio de Nitze, logró inspeccionar por primera vez en forma experimental la cavidad peritoneal de un perro.

En 1910 Jacobaeus, fue el primero en describir lo que él Ilamó "Laparoscopia" en seres humanos, utilizando un Citoscopio.

Posteriormente Nordentoeft, con el Peritoneoscopia para uso ginecológico logró por vez primera observar y fotografiar el tracto genital femenino por este medio, en el año 1912.

Desde el trabajo de Orndoff el procedimiento es conocido en Norte América como Peritoneoscopio.

En Estados Unidos de Norte América, Ruddock informó de su experiencia con 500 exámenes practicados con un instrumento ideado por él mismo; constaba de un sistema óptico, iluminación por incandescencia, aguja separada para neumoperitoneo; se po- día tomar biopsia y hacer hemostasia por electrocoagulación.

El procedimiento despertó mucho interés en su época; se publicaron varios informes donde se mostraba especialmente el valor del método en el diagnóstico temprano del embarazo ectópico. Con el instrumento ideado por Ruddock, se pensó en practicar esterilización tubárica, pero nunca se llevó a cabo.

Inicialmente la Laparoscopia usada como método diagnóstico fue propuesta como técnica de esterilización tubárica por A. T. Anderson en 1973 y luego descrita por Power y Barnes en la Universidad de Michigan en 1941.

El interés por la Endoscopia Ginecológica aumentó notoriamente a partir de 1944 cuando Decker describió la técnica de la Culdoscopia.

Palmer en Europa continuó trabajando en el desarrollo de la Laparoscopia, apreciándose mejor aceptación del procedimiento gracias a las molestias y complicaciones mínimas del mismo, lo cual se logró con la modernización del equipo inicial.

Desde esta época la Laparoscopia en Ginecología ha tenido un gran auge, demostrado por el interés de me-

* Profesor Asistente. Facultad de Medicina Universidad Nacional. 
jorar técnicas e instrumental utilizado; una mayor aplicación se ha visto en la esterilización tubárica según lo demuestran los siguientes trabajos en orden cronológico:

Palmer en Paris informó sobre 250 casos de esterilización tubárica en 1947. Recordó el uso de una cánula intrauterina hueca para un mejor manejo y visualización de los anexos al movilizar el útero.

Los años subsiguientes no demostraron mayor demanda de esta técnica como método definitivo de planificación familiar; esto era explicable dado su estado legal discutible, el temor a complicaciones, deficiencia del equipo y falta de experiencia en el personal que lo ejecutaba.

A principio de los años 60 el desarrollo de fuentes de luz fibrópticas "Frias", con un equipo auxiliar más sofisticado abrió nuevos horizontes a la Laparoscopia.

Palmer en Francia, Frangenheim en Alemania y Steptoe en Inglaterra informaron en forma amplia y entusiasta sobre la aplicación del procedimiento, en un total de 5.000 casos sin complicaciones graves.

A comienzo de la década del 70 la búsqueda mundial de mejores medios para control de la natalidad, ha estimulado el interés por la preparacóin del personal e investigación para realizarlo; así mismo se ha despertado un interés público en la aceptación de este medio en la planificación familiar. Actualmente se conocen estudios de médicos que han trabajado en este campo, sus casuísticas son muy significativas; entre ellas merecen mencionarse las siguientes:

760 casos informados por Liston y colaboradores de Escocia en 1970.
910 casos de Jordan y colaboradores de Inglaterra en 1971.

810 comunicados por Davidson y Donald de Escocia en 1972.

3.600 casos comunicados por Wheeless y Thompson en Baltimore U.S.A., durante los años 1968 a 1972.

En nuestro medio se comenzó a utilizar la Laparoscopia en Ginecología tanto con fin diagnóstico como de esterilización tubárica a comienzos del 73 siendo los primeros en aplicarla los doctores Pulido y Briceño en la Clínica de Planificación Familiar "Profamilia".

En el Instituto Materno Infantil "Concepción Villaveces de Acosta" de Bogotá, los doctores López E., Cáceres A. y Muñoz G. iniciaron el servicio de Laparoscopia a partir de Diciembre del 73 en el Departamento de Ginecología y Obstetricia en esta institución, previo entrenamiento realizado con personal médico del hospital John Hopkins de Baltimore.

Por esta misma época otro grupo de médicos del Hospital de Girardot están trabajando en este mismo campo.

Hoy día el servicio de Laparoscopia forma parte de los departamentos de Ginecología y Obstetricia de un gran número de hospitales universitarios y regionales clase $A$, beneficio logrado a través de la Corporación Centro Regional de Población de Bogotá y la Asociación Colombiana de Facultades de Medicina.

\section{Equipo:}

Los Laparoscopios pueden identificarse de diferentes maneras:

Así unos llevan el nombre del inventor, del distribuidor o un nombre comercial asignado; por ejemplo La- 
paroscopio de Palmer, Laparoscopio Fibrótico de Wolf, Laparoscopio Fibrótico de A.C.M.I.|

Actualmente existe una diferencia adicional entre los Laparoscopios para el método de dos incisiones y los Laparoscopios operatorios para el de una sola incisión.

Los instrumentos necesarios incluyen un sistema de control de presión y flujo de bióxido carbónico, una fuente luminosa fibróptica, un conductor fibróptico de luz, Laparoscopio fibróptico de acero inoxidable, trócar y cánula para introducción, aguja de neumoperitoneo. Los instrumentos auxiliares útiles incluyen pinzas de horadación para biopsia de Palmer, pinzas corrientes para biopsia, pinza para electrocoagulación, placa para conexión a tierra, pedal para electrocoagulación; algunos disponen de una aguja para neumoperitoneo con un resorte ("Verres") con ella se disminuye el peligro de una lesión intestinal al introducirla.

Mirando a través de un Laparoscopio común el cirujano puede observar los órganos abdominales y pélvicos; si desea hacer un procedimiento quirúrgico (biopsia, cauterización etc.), necesitará de una segunda incisión para introducir a través de ella los instrumentos requeridos (pinzas, cauterios, etc.).

En la actualidad se cuenta con Laparoscopios que requieren para su uso solo una incisión; entre ellos se cuenta el Laparoscopio Operatorio de Eder modificado. Este consta esencialmente de una caja metálica portátil que tiene las siguientes dimensiones: $41 \mathrm{cms}$. de alto, $23 \mathrm{cms}$. de ancho y $64 \mathrm{cms}$. de largo; en su interior se encuentra un tanque para almacenamiento del $\mathrm{CO}_{2}$ con capacidad de 5 litros; contien manómetro y registro para fluído de gas, fuente de luz, insuflador, electrocauterio, conexión a tierra y ventilador. Aparte está el Laparoscopio propiamente dicho de acero inoxidable cuyo ocular puede estar al extrem odel mismo o en ángulo recto.

Este equipo de Laparoscopio está completamente autoabastecido requiriendo solo de suministro externo de $\mathrm{CO}_{2}$, y de una entrada de energía que puede ser de 110 a 220 voltios. Las ventajas de este Laparoscopio Operatorio entre otras serían, que al eliminar la segunda incisión reducen en parte el tiempo de la operación, las quemaduras locales, así como el peligro de una perforación visceral; permite el empleo de anestésicos locales o simple analgesia. Tiene sin embargo algunas desventajas como ser, el desplazamiento que se presenta de las pinzas accesorias al mover el Laparoscopio; en igual forma la manipulación de las diferentes estructuras pélvicas está muy restringida con esta clase de equipo, que cuando se introduce elementos accesorios a través de una segunda incisión.

En nuestro servicio es el equipo que estamos usando. No disponemos de pinzas auxiliares para biopsia, ni tenemos facilidad para adaptarle equipo fotográfico; elementos indispensables para futuros trabajos.

\section{TECNICA DE PROCEDIMIENTO}

\section{Preoperatorio}

En nuestro servicio se tienen establecidas las siguientes normas:

Las pacientes remitidas para practicarles Laparoscopia con fin diagnóstico, deben ser enviadas con una hoja de solicitud, donde el médico tratante anotará los datos positivos del motivo de consulta, los hallazgos del examen practicado, su impresión diag- 
nóstica y lo que desea se aclare por este método.

A las pacientes que llegan al servicio solicitando esterilización tubárica se les llena un formulario especialmente elaborado para tal fin, donde está consignada la edad de los cónyuges, paridad, hijos vivos, sexo de los mismos, uso previo de anticonceptivos, última regla, último parto o aborto, antecedentes personales importantes como afecciones cardio-pulmonares y cirugía previa; así mismo deben presentar una solicitud y autorización firmada por el esposo o compañero. Posteriormente se les practica un examen pélvico con el fin de descartar patología ginecológica o la presencia de un embarazo.

Una vez se considera la paciente apta para practicarle el procedimiento, se le aconseja que la noche anterior a la intervención haga una cena ligera y se aplique un enema evacuador; a la mañana siguiente hacer un baño general con especial atención sobre la región abdominal y en particular el área umbilical. Se le permite un desayuno líquido temprano, debe llegar al hcspital a las 7:30 a.m., en este momento se le administra un sedante suave por vía oral como premedicación. Previo cambio de su ropa de calle por una bata quirúrgica, evacuación espontánea de vejiga, la enferma es llevada a la sala de Laparoscopia que funciona cerca del área quirúrgica sin estar involucrada en ella.

\section{Preparación en la sala}

Se coloca la paciente sobre la mesa quirúrgica en posición de Litotomía dorsal, con la región glútea $10 \mathrm{cms}$. aproximadamente fuera de la mesa, para poder manipular el útero en la dirección deseada con la cánula de insuflación. Se aplica enseguida una perfusión lenta de dextrosa al $5 \%$ en A.D. por V.I.; a través del catéter del suero se inyecta lentamente 100 mgrs. de Meperidina y 10 mgrs. de Diazepan $\circ 2 \frac{1}{2}$ cc. de una mezcla de Fentanyl y Droperidol (Talamonal) para producir analgesia. Acto seguido se lava con agua y jabón el abdomen, en especial el área umbilical y periumbilical para colocar luego alcohol yodado.

Posteriormente con la ayuda de un espéculo vaginal, se toma transversalmente el labio anterior de cuello uterino con una pinza de Jacobs y se introduce en el canal endocervical una cánula de Rubins fijándose esta a la pinza cervical; en esta forma se permite manipular los órganos pélvicas en caso de ser necesaria una mejor visualización de ellas.

El cirujano quirúrgicamente vestido cubre la paciente con un campo estéril diseñado en tal forma que deja al descubierto la zona periumbilical.

\section{INTERVENCION}

\section{Anestesia}

La técnica de una sola incisión se puede realizar con la analgesia administrada previamente a la paciente y aplicación local de unos $10 \mathrm{cms}$. de Xilocaína al $2 \%$ sin epinefrina en forma intradérmica y subcutánea en la región infraumbilical.

Los que usan la técnica de 2 incisiones prefieren el uso de anestesia general con gases no explosivos.

\section{Incisión}

Con 2 pinzas de campo se sujeta la curvatura inferior del ombligo y con bisturí se hace una incisión transversa de $1 \mathrm{~cm}$. que abarca piel y tejido celular subcutáneo. En caso de existir una hernia umbilical o ci- 
catriz quirúrgica mediana la incisión se hará en el primer caso más baja o lateralmente en el segundo.

\section{Neumoperitoneo}

La introducción de un gas en la cavidad abdominal es esencial para la Laparoscopia. El más utilizado hoy día es el $\mathrm{CO}_{2}$. Algunos médicos no lo emplean por temor a desencadenar arritmias cardíacas en la paciente por absorción excesiva de éste; prefieren el óxido nitroso por ocasionar menos cambios en el equilibrio ácido-básico de la sangre; el inconveniente mayor de este gas aparte de su costo es el peligro de manejar el equipo de cauterización por facilitar aquel la combustión, además su absorción es más lenta. En otras partes utilizan el aire por su bajo costo y fácil adquisición; pero con él se aumenta el peligro de una embolia gaseosa.

El neumoperitoneo se logra con la inserción de las agujas de Touhy, o de Verres a través de la incisión previamente hecha; en esta forma se reduce el riesgo de puncionar vasos muy grandes o de herir el intestino. Para determinar si la aguja ha penetrado en la cavidad abdominal se retira el mandril y se coloca $2 \circ 3$ gotas de suero fisiológico o agua destilada estériles dentro de la aguja, hasta que ésta se llene; luego se tracciona hacia arriba la piel con las pinzas de campo y si el líquido desaparece de la aguja es porque ella está libre en la cavidad abdominal. Se conecta luego la aguja al tubo de gas y se introducen 2 a 3 litros de $\mathrm{CO}_{2}$ como máximo, a razón de un litro por minuto. Por razones de seguridad el $\mathrm{CO}_{2}$ indirectamente de un tanque especial de distribución. Durante el paso del gas se debe controlar el registro del fluído y presión a que éste pasa; igualmente se observará la distensión y el timpanismo uniforme del bajo vientre y ausencia de enfisema en región púbica; esto indicará que el $\mathrm{CO}_{2}$ está llegando en forma correcta a la cavidad abdominal.

\section{La Laparoscopia}

Luego de haber establecido el neumoperitoneo se coloca la paciente en pasición de trendelemburg, con una inclinación de 20 a $30^{\circ}$. Esto permite que las asas intestinales, y el epiplón mayor, se aparten de la pelvis permitiendo así la visualización de los genitales internos.

Se retira la aguja de Touhy y se introduce por la misma incisión el trócar con la cánula; primero subcutáneamente en dirección horizontal en un trayecto de un $\mathrm{cm}$., y luego perpendicularmente hacia la cavidad peritoneal. Con esta forma de pasar el trócar describiendo una $Z$ se reducen los peligros de lesionar órganos nobles. Se procede luego a retirar el trócar y se permite el escape de una pequeña cantidad de $\mathrm{CO}_{2}$, para estar seguro de la posición intraabdominal del instrumento.

Antes de utilizar el Laparoscopio es aconsejable colocar la punta del mismo en solución salina caliente, en esta forma se previene el empañamiento del lente al penetrar a una. cavidad de temperatura mayor a la existente en el ambiente. Hecho lo anterior se introduce el Laparoscopio a través de la camisa del trócar y se une el cable fibróptico a la fuente de luz. Si vamos a utilizar la pinza de coagulación o de biopsia ésta se coloca al Laparoscopio en este mismo momento y se hace la conexión con la fuente de energía. Debemos cerciorarnos nuevamente si estamos dentro de la cavidad abdominal; para esto mirar a través del ocular, en caso positivo lograremos visualizar estructuras intra-abdominales como asas in- 
testinales, epiplón etc. Se introduce luego un poco más el Laparoscopio para visualizar los órganos pélvicos. Si la lente se empaña durante el procedimiento, se puede tocar el instrumento con el fondo del útero $u$ otro órgano, en esta forma se transmite a ella la temperatura corporal y se evita la condensación.

\section{Finalización de la intervención}

Terminada la exploración o el procedimiento quirúrgico se retira el Laparoscopio sin la camisa; ésta se deja unos segundos más para permitir el escape de la mayor cantidad posible de $\mathrm{CO}_{2}$ intra-abdominal, evitando en esta forma molestias posteriores a la paciente. Se retira luego la cánula, se coloca un punto subcutáneo en el sitio de la incisión con el catgut simple 00 y se cubre con un pequeño apósito.

\section{CUIDADOS POST-OPERATORIOS}

La paciente sale en camilla 0 por sus propios medios cuando se usa Neurolepsia (Talamonal), a la pieza. $\mathrm{Se}$ controlan signos vitales, presencia de dolor abdominal intenso, molestias respiratorias especialmente. Cuatro a seis horas si un control médico es satisfactorio se le permite abandonar el hospital. Al día siguiente puede reiniciar sus labores habituales.

\section{Controles}

Rige para las pacientes a quienes se les ha practicado esterilización tubárica. Un primer control se hace a los 28 días, luego a los seis meses y al año respectivamente.

En aquellos casos en que el cirujano por dificultades en el procedimiento tenga la duda de no haber seccionado bien alguna de las trompas, es conveniente practicar una Histerosalpingografía para mayor seguri- dad. Sin embargo este examen no debe ser ejecutado antes de los tres meses de haber sido hecha la Laparoscopia, pues podría permeabilizar los muñones seccionados de la trompa. Es obvio que durante este tiempo la paciente debe usar algún método anticonceptivo.

\section{Diagnóstico ginecológico}

Se reconocerá las diferentes estructuras pélvicas especialmente útero, trompas, ovarios, ligamentos redondos, fondos de saco, vejiga y recto determinando las características normales a anormales que ellas presenten; presencia o no de procesos neoplásicos, inflamatorios, adherenciales etc. Cuando se trate de pacientes con patología ovárica si se dispone de los elementos necesarios se tomará biopsia, excepción hecha en los quistes ováricos. Si la Laparoscopia se realiza en pacientes con esterilidad tubárica, se aprovechará para realizar en el mismo acto una hidrotubación con un medio colorante (Yndigo-Carmín, Azul de Metileno, Rifamicina etc.), utilizando para ella la cánula de Rubins que previamente hemos aplicado a la paciente. En esta forma se podrá evidenciar la permeabilidad tubárica.

Terminado el procedimiento se elabora un informe con los hallazgos obtenidos y una sugerencia diagnóstica del Laparoscopista, para ser enviado al médico remitente.

\section{ESTERILIZACION TUBARICA}

En este procedimiento existe diversidad de criterios en cuanto al sitio a elegir de la trompa y la forma en que se debe realizar. Así unos autores prefieren tomar la porción ístmica, otros la región ampular y hay quienes lo hacen en la zona del pabellón. Igualmente unos solo coagulan, otros coagulan y seccionan la trompa y fi- 
nalmente otro grupo coagula, secciona y retira una porción de ella.

En el servicio seguimos la técnica del hospital de John Hopkins en Baltimore, que consiste en tomar con la pinza el istmo de la trompa uterina (más o menos a dos cms. del cuerpo) aislando esta porción de las estructuras vecinas. Una vez hecho esto se electrocoagula pisando el pedal del cauterio por unos 10 segundos, hasta lograr blanquear unos $2 \mathrm{cms}$. de trompa quedando los cabos del segmento completamente separados. Se ejecuta luego el mismo procedimiento en la trompa contralateral.

Las maniobras con el tenáculo y la cánula de Rubins puesta previamente en el cuello, facilitan al llevarlo hacia uno y otro lado de la visibilidad de las trompas para poderlas agarrar por el fórceps.

Una vez electrocoaguladas y seccionadas ambas trompas, se retira la pinza de electro-coagulación del Laparoscopio y se revisa totalmente la pelvis. El retiro de la pinza ayuda a evitar accidentes de quemaduras en asas on otras estructuras pélvicas, en el momento de la revisión. Si durante esta se encuentra algún punto sangrante en los muñones de las trompas puede electrocoagularse nuevamente.

Esta técnica parece ser la más confiable. Los riesgos de embarazos posteriores no pasan de 1/1.000 mientras que el porcentaje se eleva considerablemente en las series donde solo usan coagulación. La falla operatoria puede ocurrir cuando la coagulación ha sido incompleta y ha dejado una - las dos trompas permeables, o cuando se ha cauterizado el ligamento redondo o algún otro elemento en vez de la trompa de Falopio. En ocasiones puede suceder que la inter- vcención se realiza en pacientes previamente embarazadas; para obviar esto se aconseja que la mujer esté usando un método anticonceptivo seguro antes del procedimiento.

\section{Indicaciones}

La Laparoscopia es un elemento de ayuda muy importante en el estudio de la patología abdominal ginecológica. En ocasiones confirman los hallazgos encontrados en el examen pélvico o exámenes para-clínicos y en otros es el único medio para llegar a un diagnóstico definitivo.

Fundamentalmente se usa: en estudio de ascitis, Hepatomegalia, Ictericia, investigación de metástasis, neoplasias, Tuberculosis y con carácter de urgencia en traumatismos abdominales.

Desde el punto de vista ginecológico sus indicaciones son: malformaciones congénitas, neoplasias, procesos inflamatorios crónicos, masas discutibles en anexos, esterilidad, disgenesia gonadal, embarazo ectópico, dolor pélvico crónico sin causa aparente, endometriosis. Una indicación más reciente es la esterilización tubárica.

\section{Contraindicaciones}

Las siguientes entidades contraindican en forma absoluta el procedimiento: Afecciones cardio-pulmonares descompensadas, peritonitis agudas, múltiples adherencias que impidan obtener un buen neumoperitoneo, tumores gigantes que obstaculizan la penetración a la cavidad peritoneal, hernias voluminosas.

Son consideradas contraindicaciones relativas la cirugía abdominal previa, cicatrices abdominales extensas, obesidad exagerada. 


\section{Complicaciones}

La mayor parte de los Laparoscopistas están de acuerdo que el procedimiento tiene una tasa de morbilidad muy baja. Las series revisadas más numerosas que corresponden a la esterilización tubárica, dan un porcentaje que varía entre el $1 \%$ al $6 \%$.

Las complicaciones mayores más frecuentes son el enfisema subcutáneo - mediastinal, problemas cardíacos y respiratorios, perforación de órganos, quemaduras y hemorragia. Ocasionalmente embolia gaseosa.

\section{Conclusiones}

Después de esta revisión sobre la Laparoscopia considero que los ginecólogos contamos con un arma muy efectiva, que usada en los casos que tiene cabida representa una ayuda más en el estudio paraclínico.

Su uso debe rutinizarse en los servicios ginecológicos; en esta forma se ofrecerá mayores garantías a la paciente: más exactitud en el diagnóstico, ahorro de tiempo, costo y molestias ocasionadas por exámenes paraclínicos en muchos casos innecesarios y de resultados poco confiables (Histerosalpingografía, Ginecografía, etc.).

Debe ser interés de los departamentos de Gineco-Obstetricia adiestrar al personal docente en estas disciplinas para que ellos a su vez puedan transmitirlas a los especialistas en formación.

De este punto de vista en nuestor servicio se está cumpliendo la premisa anterior y es así como hoy día aparte de los dos docentes que manejan el servicio, tenemos cuatro profesores ya entrenados. Los residentes rotan por el servicio a nivel de R-2 durante un mes. En este tiempo tienen oportunidad de practicar bajo supervisión el procedimiento, en las pacientes que nos llegan para ser sometidas a él ya sea con fines diagnósticos o esterilizantes.

\section{Conclusions}

After this revision of laparoscopy, it is my belief that we the gynecologists have a very effective tool available which, if used in the cases where applicable, represents another aid for the para-clinical study.

It use should be routinary at the gynecological services; in this manner it would be possible to offer greater guaranties to the patient, such as exactness in diagnosis, time saving, cost saving and less discomforts caused by para-clinical tests which in many cases are unnecessary and have little trustful results (hysterosalpingography, gynecography, etc.).

The Gynecological Obstetrical Departments should become interested in the training of educational personnel of this field in order that they in turn may train specialists.

From this point of view, our service is implementing the aforesaid task and at this time, aside from the two students in charge of the service, we have four professors trained. The residents are shifted by the service at an R2 level during one month. During such time, they have an opportunity to apply the system under supervision in those patients who come for its application either for diagnosis or sterilization purposes.

\section{BIBLIOGRAFIA}

1 ANDERSON, E. T. "Peritoneoscopy". American Journal of Surgery. 35: 36-39, 1937.

2 GARATY, R. Y KARIS, J.: Blood gas studies during laparoscopy under general anesthesia. Anesthesiology; 30: 463, 1969. 
3 BOER, C. H. and DUNSTER, G. "Tubal electrocoagulation and assessment". American Journal of Obstetrics and Gynecology. 3 (7) 979-983, Diciembre 1\%, 1971.

4 COHEN, MELVIN, R., TAYLOR, MARSHALL BERMENT, and KASS, MARIN, B. "Internal tubal Sterilization via Laparoscopy". American Journal of Obstetrics and Gynecology, 109 (5) 794-809, Marzo 1\%, 1971.

5 COHEN, M. R.: Culdoscopy vs. Peritoneoscopy. Obstet. Gynec. 31: 310, 1968.

6 FEAR, R. E.: Laparoscopy: Avaluable aid un Ginecology diagnosis. Obstet. Gynec. 31 : 297, 1968.

7 COLDITCH, IRA, M. "Laparoscopy: Advances and Odvantages, Fertility and Sterility". 22 (5): 306-310 May. 1971.

8 JORDAN, J. A., EDWARD, R., LOGAN, PEARSON J. and MASKWERY P. J. K. "Laparoscopic Sterilization and Follow-Up Histerosalpingogram". The Journal of Obstetrics and Gynecology of the British Commohealth. 78: 440-466, Mayo 1971.

9 LISTON, W. A., BRADFORD, W., DOWNIE, J. and KERR M. G. "Female Sterilization by Tubal Electro-Coagulation Under Laparoscopic Control". The Lancet. 382-383. Feb. $21,1970$.

10 MAHENDRA, G. H. ARRONET: Procedimientos y Métodos diagnósticos para valorar los órganos genitales femeninos, con referencia especial a la esterilidad. Clin. Obstet. Gynec. 2: 1-99, 1972 .

11 MILTON, CAMPOS, MONTOYA: Valor de la Laparoscopia en las enfermedades de la cavidad peritoneal. Rev. Fac. Med. U.N. Vol. XXXVIII, No $3-72$.

12 NEUWINTH, R. S.: Laparoscopy. Clin. Obstet. Gynec. 12: 514-26, 1969.

13 ORNDOFF, B. H.: Hhe Peritoneoscope in diagnosis of diseases of the abdomen. Am. Surg. 13: 754, 1920.

14 PENT, D., Laparoscopy: Its role in private practice. Am. Journal of Obstetrics and Gynecology. 113 (4): 459-468.

15 PETERSON, E. P. and BEHRMAN, S. J. "Laparoscopic Tubal Sterilization". American Journal of Obstetrics and Gynecoolgy. 110 (1): 24-31, Mayo 19, 1971.

16 STEPTOE, P. C. "Problems of Laparoscopic Sterilization". The Lancet. 1: 1115, Mayo 20, 1972 .

17 TELINDE: Culdoscopia. Ginecología operatoria. 3a. ed. Pág. 69, 1966.

18 WHEELESS, C., CLIFFORD, R. Jr. Cutpatient Laparoscope Sterilization Under Local Anesthesia, Obsterics and Gynecology 39 (5) : 767-770, Mayo, 1972.

19 WHEELESS, C., and THOMPSON, B. "Laparoscopic Sterilization". Review of $3.600 \mathrm{Ca}$ ses. Obstet. and Gynecology. 42: 751-758, 1973. 